\title{
N $93-15511$
}

COMBLNING RAY.TRACE AND DIFFRACTION ANALYSIS:

A DESIGN EXAMPLE

Tom D. Milster

Optical Sciences Center, University of Arizona, Tucson, Arizona 85721

Jeffrey P. Treptau

Cray Research, Chippewa Falls, WI 54729

\begin{abstract}
We present an example of using a combined ray trace and diffraction modeling code to simulate effects of objective-lens tilt in an optical data storage device.
\end{abstract}

\section{L.NTRODUCTION}

In some cases, neither ray-trace analysis nor diffraction analysis can give an adequate description of an optical system. The designer that is faced with the problem of analyzing such a system is forced to use a ray-trace program to determine aberrations in the exit pupil and then introduce aberration coefficients into a diffraction model that simulate the propagation. We find this approach rather awkward, especially if complicated aberrations are present. Our approach is to integrate a diffraction analysis and a ray-trace description of an optical path into one program.

Our design is taken from a data storage application, where we must analyze the effects of objective-lens tilt. The optical path is shown in Figure 1. A diode laser beam is collimated, and it is circularized with an anamorphic prism pair. The beam then passes through a partially polarizing beam splitter, (PPBS), which passes $70 \%$ of the irradiance polarized in the $p$ direction and reflects $100 \%$ of the $s$ polarization. The transmitted beam then reflects off of the tuming mirror into a high numerical aperture (NA) objective lens. The objective lens is usually constructed as a molded bi-aspheric element. The light is then focused down to the disk plane through a cover plate. At the recording layer, there is a complicated interaction berween the laser spot and the medium. The disk usully has a groove structure, which means that the analysis must include interaction with a periodic phase object. If the medium is a magneto-optic film, the analysis must include effects of polarization rotation upon reflection. After reflection, the beam is recollimated and deflected into the servo and data detection optics.

This application requires many types of analysis. Since we are using a laser diode, we must consider the type of beam that a laser diode generates with a physical-optics description. Interaction with the medium also requires a physical-optics description. Magneto-optic media require polarization effects to be simulated. A ray-trace analysis is necessary to quantify the aberrations introduced by the tilted objective lens.

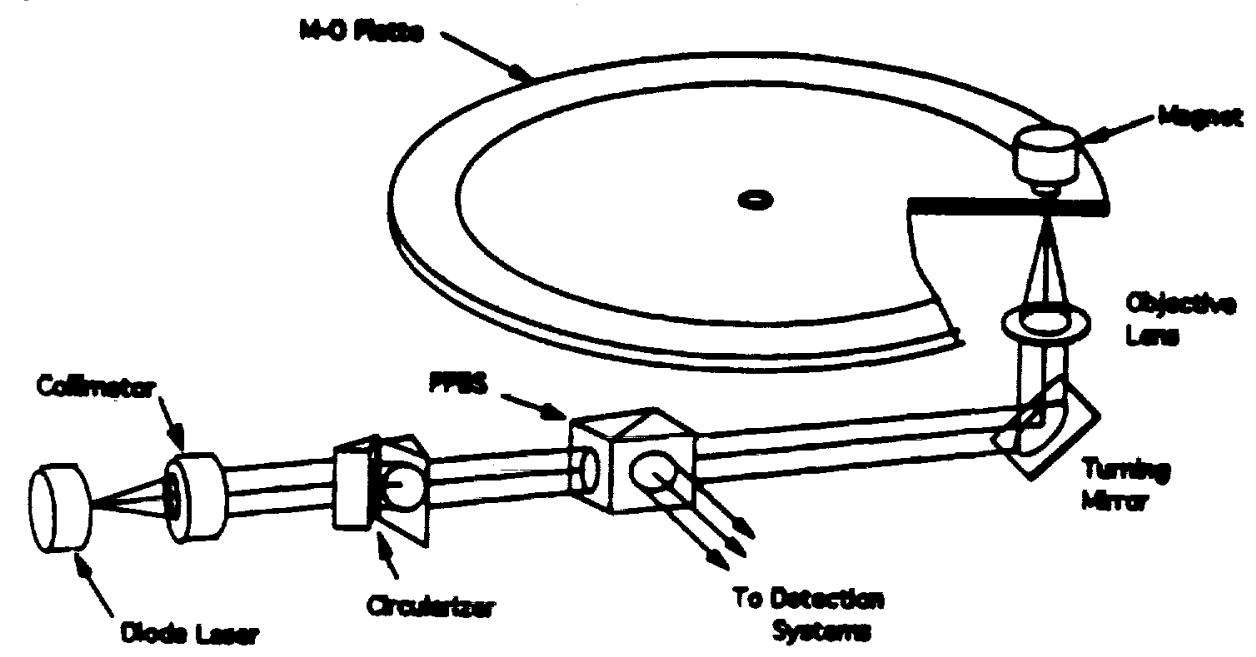

Figure 1. Layout of an optical data storage device. 


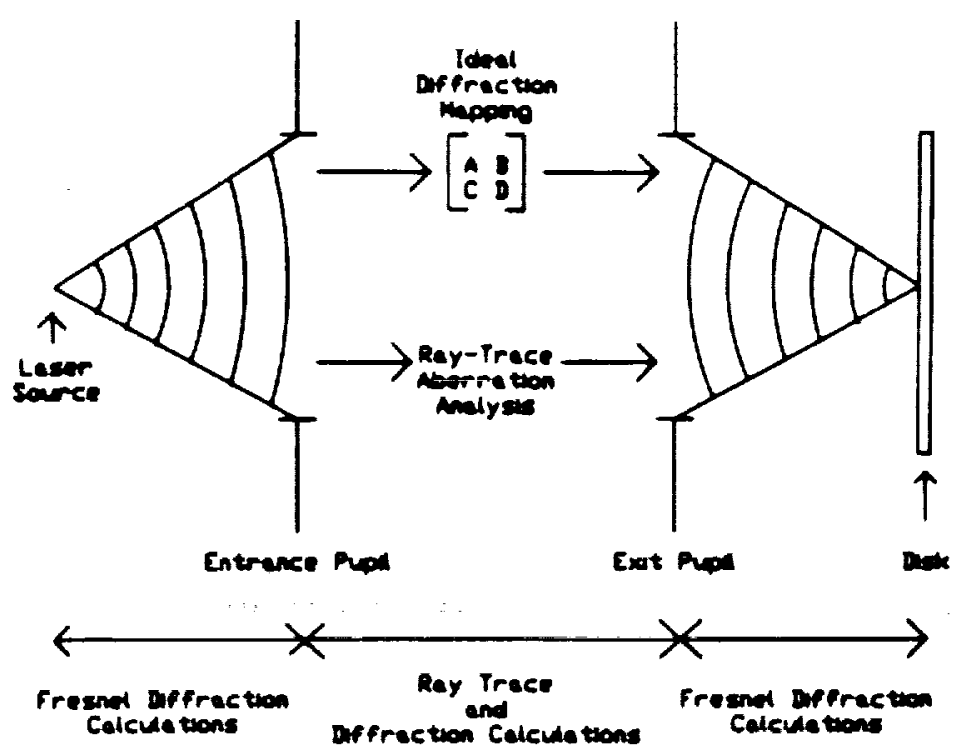

Figure 2 Diagram of entrance pupil to exit pupil mapping technique.

In a previous publication (1) we have described how to analyze these effects. Our model uses a ray trace from the object to the image plane and a scalar diffraction propagation to simulate physical optics phenomena. There are commercial modeling codes, like the OLAD analysis pactage (2), that come close to meeting all of our requirements. However, at the time of this writing, we are working with a code that has been developed in our laboratory. In this paper, we review basic principles of the model and study the effects of objective lens tilt at several planes in the optical system.

\section{THEORY}

Modeling of the optical system is accomplished in a two-pronged approach, as shown in Figure 2 To begin, the wavefront trom the facet of the laser diode is propagated to the entrance pupil of the system with scalar diffraction. Next. we calculate a paraxial system quantifler, the $A B C D$ matrt, which describes propagation from the entrance pupil to the exit pupil. Once the system ABCD matrtx has been found, that information is placed into Huygens' integral for wave propagation (3). To this point, we bave a physical-optios description of the teld in the exit pupil.

The aberrations in the optical system must now be considered. We start with a point source at the laser diode and trace a matrix of rays to the disk plane. The optical path distance (OPD) traversed by each ray is accumulated during the trace. Each ray is then reverse traced to the exit pupil of the system. Ray sampling is chosen so that each ray is identified with a single pirel in a square matrix representing the exit pupil. The square matrix of sample points is the same as for the difrnction propagation discussed in the previous paragraph.

The ideal difraction wavetront and the OPD in the exit pupil due to aberrations are combined to give the total exit-pupil wavefront. The resulting phase is just the addition of the two component phase fronts on a pirel-by-pixel basis. The amplitude of the beali at the exit pupil is given by the ideal diftraction propagation of the electric field amplitude incident on the entrance pupil.

The wavefront represented at the exit pupil is propagated to the disk using scalar diffraction calculations. After interaction with the medium, the reflected beam is propagated back through the optical system in a similar manner.

\section{MODELING RESULTS}

This model is implemented on a 486 computer that runs at 33MHZ. The modeling environment is the MATLAB programming language (4). MATLAB is an interactive mathematics programming environment that allows a user to 
Table I

\begin{tabular}{|c|c|c|c|c|c|c|c|c|c|c|c|}
\hline${ }_{\operatorname{lom} 2 d i n t}=[\ln x$ & $\begin{array}{l}\text { THICR } \\
2590620.3\end{array}$ & $\begin{array}{l}\text { INDEX } \\
\text { NR }\end{array}$ & $\begin{array}{l}\text { OWM } \\
\text { OW }\end{array}$ & $\begin{array}{l}\text { DECENX } \\
0\end{array}$ & DECENY & $\operatorname{Tn}_{0} \operatorname{Tx}$ & ${ }_{0}^{\pi L T}$ & ${ }_{0}^{\text {CONKC }}$ & $\begin{array}{l}0 \\
0\end{array}$ & $\begin{array}{l}\mathrm{E} \\
0\end{array}$ & F \\
\hline 12576 te-3 & $250-3$ & SFY & $5.210-3$ & 0 & 0 & 0 & 0 & 0 & 0 & 0 & a. \\
\hline $10.473-3$ & $3.556+3$ & SRII & 5.36 .5 & 0 & 0 & 0 & 0 & 0 & 0 & 0 & 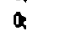 \\
\hline $.162137-3$ & $2(6300428.3$ & NR & $565 e-3$ & 0 & 0 & 0 & 0 & 0 & 0 & 0 & 0. \\
\hline $\ln t$ & {$[x-3$} & AlR & 4.3.3 & 0 & 0 & 0 & 0 & 0 & 0 & 0 & 0 \\
\hline Int & $\sin 3$ & BX7 & $1270-3$ & 0 & 0 & 0 & 0 & 0 & 0 & 0 & 0 \\
\hline int & $\tan 3$ & AIR & $127-3$ & 0 & 0 & 0 & 0 & 0 & 0 & 0 & o. \\
\hline Inf & le-3 & CRYSTAL & $127-3$ & 0 & 0 & 0 & 0 & 0 & 0 & 0 & 0. \\
\hline inf & $19.27318 k .3$ & UR & $127 e-3$ & 0 & 0 & 0 & 0 & 0 & 0 & 0 & 0. \\
\hline 25.3864 - 3 & $3.5 e-3$ & SK11 & $15-3$ & 0 & 0 & 0 & 0 & 0 & 0 & 0 & 0. \\
\hline$-17.1778+3$ & 203 & SFS & 15.3 & 0 & 0 & 0 & 0 & 0 & 0 & 0 & 0 \\
\hline$-6.121-3$ & $37.360000-3$ & AR & $15+3$ & 0 & $\underline{0}$ & 0 & 0 & 0 & 0 & 0 & 0 \\
\hline Int & $37.673606-3$ & AR & 2203 & 0 & o & 0 & 0 & 0 & 0 & 0 & 0 \\
\hline $69.121-3$ & $2-3$ & SFI & $15 x-3$ & 0 & 0 & 0 & 0 & 0 & 0 & 0 & 0 \\
\hline $17.1375 .-3$ & $3.5-3$ & Sxil & $15-3$ & 0 & 0 & 0 & 0 & 0 & 0 & 0 & 0 \\
\hline$-25.58<-3$ & 25.202651e-3 & AlR & $15+3$ & 0 & 0 & 0 & 0 & 0 & 0 & 0 & 0 \\
\hline $\ln$ & $1.3+3$ & $\mathbf{N R}$ & $4.32-3$ & 0 & 0 & 0 & 0 & 0 & 0 & 0 & D. \\
\hline $\begin{array}{l}287396-3 \\
.3243076-3\end{array}$ & $\begin{array}{l}2: 27607-3 \\
23 \times 264-3\end{array}$ & $\begin{array}{l}\text { SEIG } \\
\text { ARR }\end{array}$ & $420-3$ & 0 & $\begin{array}{l}0 \\
0\end{array}$ & $\begin{array}{l}\text { opidice } \\
\text { opidise }\end{array}$ & $\begin{array}{l}0 \\
0\end{array}$ & $\begin{array}{l}0 \\
0\end{array}$ & \multicolumn{3}{|c|}{ 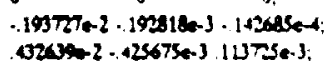 } \\
\hline Int & $L 20+3$ & PST & $4-3$ & 0 & 0 & opiviso & 0 & 0 & 0 & 0 & 0 \\
\hline Is & 0 & AlR & 43 & 0 & 0 & oppiso & 0 & 0 & 0 & 0 & 0 \\
\hline In & 0 & $\mathbf{N R}$ & $20-3$ & 0 & 0 & 0 & 0 & 0 & 0 & 0 & 이: \\
\hline
\end{tabular}

perform a variety of calculations, including those necessary for the scalar diffraction model of light propagating through the system.

The setup parameters are entered through a text file that is edited before beginning the master subroutine. Input parameters for laser diode beam widths, beam quality, wavelength, etc., are placed in the text file. Other parameters, like polarization characteristics, media format, data patterns, etc., are also placed in the tent file. The optical system is entered in the text file as matrix, as shown in Table I for the beam path from laser to disk. The input format for the optical system closely resembles the format used in other ray-trace codes. The optical system consists of the collimator, a beam splitter, a relay leas pair, and a NA $=0.55$ objective lens. The return path to the servo detectors looks very similar, except that the path is reversed and a lower NA lens is used to focus down onto the detectors. The operating wavelength for this ecample

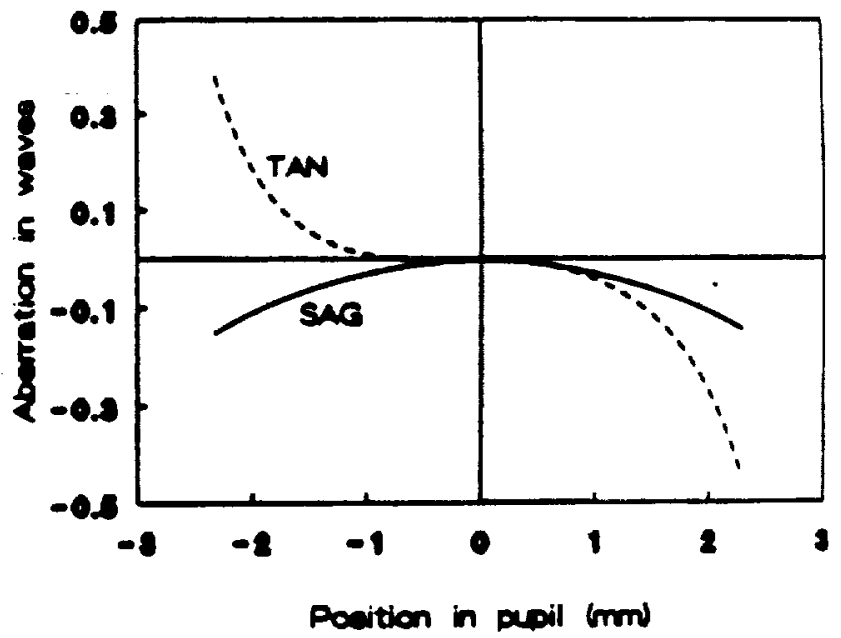

Flgure 3. Sagittal (SAG) and tangential (TAN) wave fans for an objective lens tilted $0.50^{\circ}$. is $780 \mathrm{~nm}$. The stop is located at the objective lens and has a diameter of $4.0 \mathrm{~mm}$.

Ideal diffraction propagation is accomplished irst. Next, we calculate the OPD distribution due to the ray trace. The field in the exit pupil is calculated trom the beam emitted by the laser diode and the system ABCD matrix. A profile of wavefront for a lens tilted $0.5^{\circ}$ is shown in Figure 3, which indicates a combination of coma and astigmatism in the sagittal (SAG) and tangential (TAN) wave hans. Next, the diffraction to the disk plane is performed. Figure 4A-C displays spot field distributions for $0^{\circ}, 0.25^{\circ}$, and $0.50^{\circ}$, objective lens tilt, respectively. The primary aberrations are coma and astigmatism. The teld distribution is multiplied by the appropriate disk matrices. An example of a phase matrix representing a groove structure on an optical disk is shown in Figure 5. Propagation back to the detector plane results in the irradiance distributions shown in Figures $6 \mathrm{~A}-\mathrm{C}$ for objective-lens tilts of $0^{\circ}, 0.25^{\circ}$, and $0.50^{\circ}$, respectively. The small bumps located on each side of the central lobe are due to diffaction from the groove structure. Coma, because it is an odd aberration, is effectively canceled when the reflected light propagates through the objective lens. Astigmatism. because it is an even aberration, is doubled. The irradiance distribution at the detector plane can now be summed over sections of the matrix to simulate effects of bi-cell, quad-cell, or other multi-element detector. 


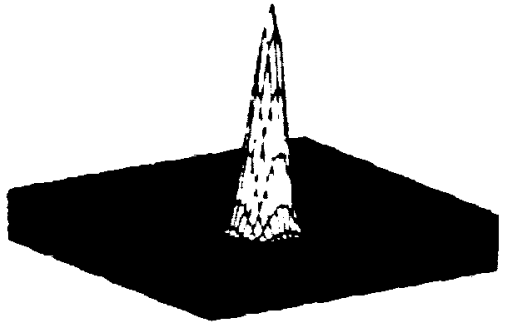

A

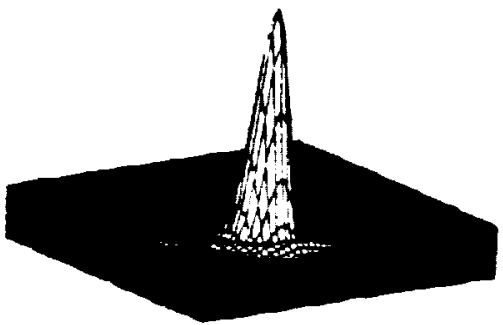

B

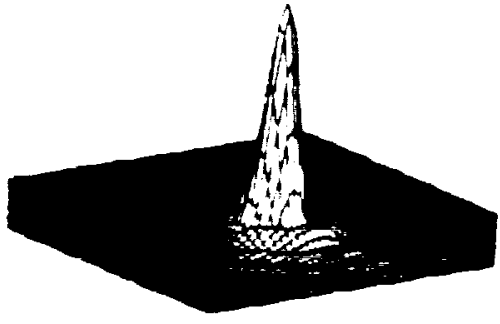

C

Figare 4. Spot irradiance distributions on the medium for objective-lens tilts of: A) $0.0^{\circ}$; B) $0.25^{\circ}$; and C) $0.50^{\circ}$.

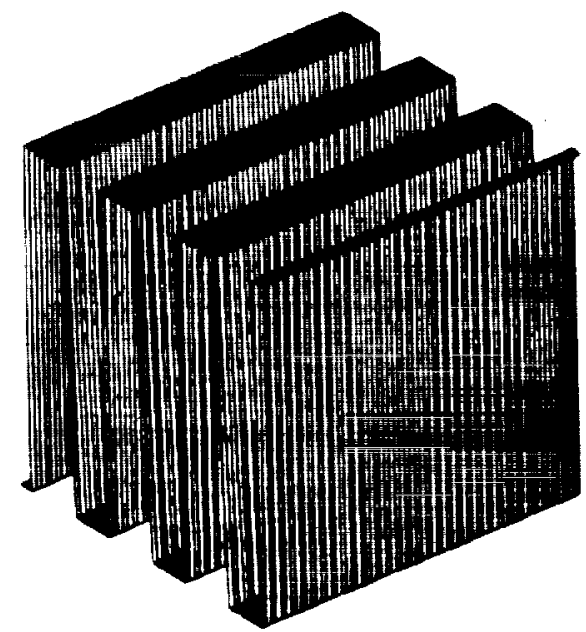

\section{SUMMARY}

We have shown an implementation of a combined ray trace and diffraction optical modeling code. Our example application was the determination of effects due to objective-lens tilt in an optical data storage device. A physical-optics description of the beam emitted from the laser diode was used, along with a physical-optics description of the laser interaction with the storage medium. Salar diffraction theory was used to propagate from plane to plane. The ray-trace analysis was used to determine aberrations due to the tilted lens. Other effects, such as polarization phenomena, can also be modeled.

Figure 5. Phase matrix representing the groove structure on the disk

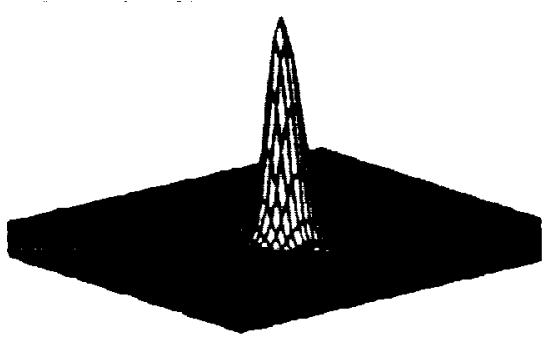

A

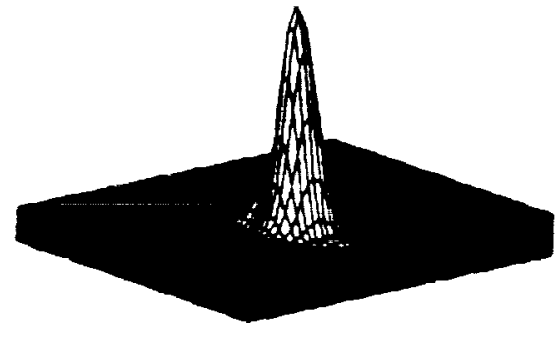

B

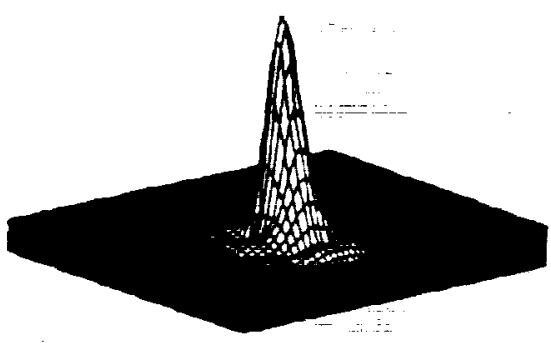

C

Figure 6. Irradiance distributions on the detector plane for objective lens tilts of: A) $0.0^{\circ}$; B) $0.25^{\circ}$; and C) $0.50^{\circ}$. 


\section{ACKNOWLEDGEMENTS}

This work was supported by NASA grant no. NGT.50579 and the Optical Data Storage Center.

\section{REFERENCES}

1. J.P. Treptau, T.D. Milster and D.G. Flagello, "Laser Beam Modeling in Optical Storage Systems," SPIE Modeling and Simulation of Laser Systems II, 1415, pp. 317-321 (1991).

2. Applied Optics Research, commercial literature (1991).

3. A.E. Siegman, Lasers, University Science Books (1986).

4. The Math Works, Inc., (1989). 
$\sigma$

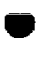

$\sigma$

$\sigma$

$\bar{\sigma}$ 


\section{APPENDIX G}

$\mathcal{U}$ 
$\sigma$ $\Rightarrow$

$\varpi$

$\sigma$

$\sigma$ 